\title{
Load-Balancing Rendezvous Approach for Mobility-Enabled Adaptive Energy-Efficient Data Collection in WSNs
}

\author{
Jian Zhang ${ }^{1,4}$, Jian Tang ${ }^{2 *}$, Zhonghui Wang ${ }^{3}$, Feng Wang ${ }^{3}$ and Gang Yu ${ }^{5,6}$ \\ ${ }^{1}$ College of Computer and Software, Nanjing University of Information Science and \\ Technology, Nanjing 210044, China \\ [e-mail: jianzhang_neu@163.com] \\ ${ }^{2}$ Department of Information, Beijing University of Technology, Beijing 100124, China \\ [e-mail: tjian001@126.com] \\ ${ }^{3}$ Department of Computer and Information Science, The University of Mississippi, Mississippi, MS 38655 USA \\ ${ }^{4}$ Engineering Research Center of Digital Forensics, Ministry of Education, Nanjing 210044, China \\ ${ }^{5}$ State Key Laboratory of Process Automation in Mining \& Metallurgy, Beijing 102600, China \\ ${ }^{6}$ Beijing Key Laboratory of Process Automation in Mining and Metallurgy, Beijing 100089, China \\ *Corresponding author: Jian Tang
}

Received December 23, 2019; revised March 5, 2020; accepted March 15, 2020; published March 31, 2020

\begin{abstract}
The tradeoff between energy conservation and traffic balancing is a dilemma problem in Wireless Sensor Networks (WSNs). By analyzing the intrinsic relationship between cluster properties and long distance transmission energy consumption, we characterize three node sets of the cluster as a theoretical foundation to enhance high performance of WSNs, and propose optimal solutions by introducing rendezvous and Mobile Elements (MEs) to optimize energy consumption for prolonging the lifetime of WSNs. First, we exploit an approximate method based on the transmission distance from the different node to an ME to select suboptimal Rendezvous Point (RP) on the trajectory for ME to collect data. Then, we define data transmission routing sequence and model rendezvous planning for the cluster. In order to achieve optimization of energy consumption, we specifically apply the economic theory called Diminishing Marginal Utility Rule (DMUR) and create the utility function with regard to energy to develop an adaptive energy consumption optimization framework to achieve energy efficiency for data collection. At last, Rendezvous Transmission Algorithm (RTA) is proposed to better tradeoff between energy conservation and traffic balancing. Furthermore, via collaborations among multiple MEs, we design Two-Orbit Back-Propagation Algorithm (TOBPA) which concurrently handles load imbalance phenomenon to improve the efficiency of data collection. The simulation results show that our solutions can improve energy efficiency of the whole network and reduce the energy consumption of sensor nodes, which in turn prolong the lifetime of WSNs.
\end{abstract}

Keywords: WSNs, Data collection, Mobile elements, Diminishing marginal utility rule.

This research was supported in part by National Natural Science Foundation (NNSF) of China (61803191,61703089), State Key Laboratory of Process Automation in Mining \& Metallurgy and Beijing Key Laboratory of Process Automation in Mining \& Metallurgy (BGRIMM-KZSKL-2018-06) and PAPD. 


\section{Introduction}

W ireless sensor networks (WSNs) have drawn significant attentions for past decade due to their pervasive applications and low deployment cost [1], which have provided practical solutions for a wide range of applications as an accessible information collection system [2,3], such as military surveillance, habitat monitoring, etc. However, designing data collection approach is a challenge, because sensor nodes are often powered by batteries with limited energy resources and if without careful design, wireless communication in data collection can energy-efficient issues and others [4-6]. For example, sensor nodes in multi-hop WSNs with MEs are utilized to continuously update the current location of Sink to maintain an accurate trajectory and balance energy consumption, which leads to more information exchange as well as more energy consumption [7]. Additionally, because data are accumulated while being collected along the path to the Sink, this will create an energy consumption bottleneck, which is further aggregated by unreliable communication and constrained resources [8]. Therefore, a critical issue in WSNs is how to balance the energy consumption of sensor nodes to alleviate these phenomena in the process of data collection.

In fact, multi-hop and long-range wireless communications exactly cost high transmission energy for sensor nodes, which tends to shorten the lifetime of network [9]. To solve this problem, two significant strategies are usually proposed to satisfy energy-efficient requirements in applications. One strategy is to introduce ME, like what proposed in [10-12] to achieve energy efficiency for data collection. The other one is to build clustering algorithm, which reduces and balances energy consumption in order to prolong the lifetime of WSNs [13].

Indeed, clustering definitely makes a network look "smaller" and extends its lifetime by reducing data transmission between nodes and the Sink [14]. However, the cluster head (CH) generally consumes more energy than cluster members due to the responsibility of collecting and forwarding data, which makes CH's energy run out more quickly. In this case, introducing MEs has been proved that it is not noly an effective approach for addressing the energy optimization issue to prolong the lifetime of WSNs but also an effective manner for organizing WSNs to achieve energy balance [15,16]. Sequentially, the participation of ME inevitably causes the problem of time delay [17], which comes to be dealt with when ME is employed as an assisted tool to improve the performance of WSNs. For example, cluster-based techniques supporting ME are more efficient for data collection [18]. However, it will take more time to collect data because ME generally moves with a slow speed and regularly stops at a prearranged location after tracing predetermined paths, which is very fatal for data collection system in terms of delivering emergency data as quickly as possible. In consequence, it is of importance to concentrate on the problem of delay problem for data collection by combination of both [19]. Additionally, the transmission model is also regarded as one of critical influence factors for the data collection in terms of the energy efficiency[20]. In fact, the energy consumption of a node increases exponentially with the growth of the transmission range, which is intuitively shown in Fig. 1. Especially, the phenomenon of energy imbalance in the cluster assuredly comes to occurrenc when some members have longer transmission ranges.

In view of above problems and inspired by concepts of Ring for WSNs in [21] as well as the network framework in [22], we build a cluster-based structure for WSNs and design RP for ME to stop and collect data from each cluster, aiming at minimizing energy expenditure efficiently. For RPs, we assume a mobile charging station is available for ME to harvest energy when ME arrives at RP, because ME generally is a battery-powered robot and usually needs 
energy supply. Nevertheless, the coordination between the charging station and MEs is beyond the research range in this paper, and we mainly focus on the optimization of RP selection to achieve the performance of cluster-based WSNs. In this paper, we also assume that MEs move on peripheries of concentric Rings as fixed trajectories.

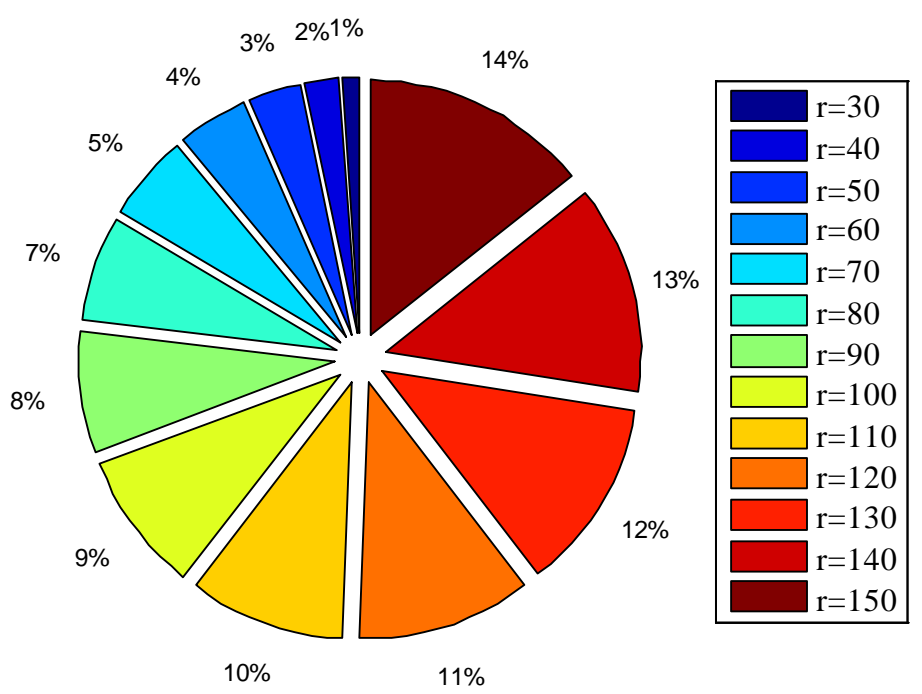

Fig. 1. Energy Consumption Proportion of Nodes with Different Radii.

Motivated by above researches and preparations, we propose the RTA which deals with the optimization of energy consumption for the data collection via integrating the DMUR into the energy consumption of the cluster. For improving the efficiency of data collection, we further propose the TOBPA to shorten the time interval of collecting data and to reduce packages for reducing energy. In this paper, we summarize our contributions as follows.

1) We design a rendezvous-based energy optimization mechanism through building the network structure and defining roles of members in cluster, which creates a framework for data collection.

2) We develop a utility function, and then propose an adaptive algorithm to search RNs based on DMUR for the optimization of energy consumption.

3) For the problem of delay caused by mobility, we come up with TOBPA to alleviate this phenomenon, which contains effectively searching RNs and consciously reducing data load for energy efficiency.

4) Extensive simulations conclude that our proposed algorithms are of effectiveness.

The rest of this paper is organized as follows: Section 2 reviews related works. Section 3 introduces our models and formulates the studied problems. Mechanisms on Rendezvous planning for data collection and MEs cooperation are presented in Section 4 and Section 5, respectively. Simulation results are presented in section 6, and Section 7 concludes the paper.

\section{Related Work}

Extending the lifetime of sensor nodes powered by batteries with limited energy resource is a challenging issue in WSNs, and extensive research achievements have been applied for energy efficiency [23-25]. In [23], Zhang proposes a directional virtual backbone to investi- 
gate the energy consumption density performance in data collecting. Other approaches [26-28], which imploy the mobility of ME as an effective technique to address the energy efficiency, are proposed for the energy-efficient utilization. However, none of them resolve the energy problem of multi-hop data transimission which partial nodes deplete energy too fast due to data gathering near the Sink or ME, although all of them make the performance of WSNs improve to some extent. From this perspective, other resolutions need to be worth exploring. At present, two strategies can be applied to enhance the energy efficiency: 1) using hierarchical routing based on clustering and 2) by introducing MEs.

The cluster-based technology is widely used as an efficient approach for organizing the networks and achieving the energy efficiency in [21,29]. In general, clustering in [30] is proposed by providing a fair distribution of clusters in the network, which elects cluster head $(\mathrm{CH})$ in turn among members to guarantee load balance and energy consumption optimization. In [15], several variations of clustering techniques are applied to enhance the performance of WSNs for data collection. The authors in [31] propose the hierarchical clustering algorithm to achieve a balance between energy consumption and the quality of service. Different from other energy-efficient routing schemes, the main target in [32] is to research adaptive cluster-based routing algorithms for improving the energy efficiency and data transmission performance. Nevertheless, $\mathrm{CH}$ always run into the predicament of energy bottleneck due to the heavy communication with other $\mathrm{CHs}$ or the Sink. In addition, frequent $\mathrm{CH}$ elections incur a massive exchange of information, which further aggravate the network burden.

In practical applications [33,34], ME keeps moving around within the region of interest for collecting data. Explicitly, efficient data-forwarding strategies are proposed with ME which visits RPs to gather data from sensor nodes. In [35], Xing et al. offer an efficient rendezvous algorithm to determine ME's trajectory by a subset of sensor nodes serving as RPs, which collects data originated from sources and transfer them to ME. Authors in [36] also utilize RPs to select an energy-efficient mobile-sink path to address energy challenge problems. However, it is difficult for sensor nodes serving as RPs to fundamentally solve the problem of network load balance, which directly determine the lifetime of WSNs. In this sense, introducing MEs can mitigate embarrassment from a sengle ME. So, MEs in [37] are coordinated to establish a connection between source node and base station in order to minimize traveling distance and hop counts of data transmission. For data transmission in the dynamic network, Authors in [38] propose an energy-efficient cluster-based dynamic route adjustment approach with MEs aiming to minimize the route reconstruction cost of sensor nodes.

Actually, a single ME can cause data latency owing to low speed movement. In [39], Liu formulates the latency problem according to rendezvous-based mobile data collection and researches local data aggregation to shorten it. Different from Liu, authors in [40] theoretically investigate the strategy that minimizes the total expected flooding delay with a mobile sink by considering all the possible traveling orders. In [41], the problems of latency from mobility are referred by introducing ME and assigning RPs, which reduces the number of multi-hop transmissions to balance energy consumption. Although a single ME strategy take a full consideration in terms of latency for data collection, it is not suitable large-scale WSNs. What's more, collecting real-time data is another challenge that the mobility strategies have to confront. Under the condition of arbitrary numbers of ME, Shusen Yang [42] focuses on low-delay opportunistic data collection in WSN-MEs with general network topologies. 


\section{Problem Formulation}

\subsection{Network Model}

The energy consumption follows the popular model given in [43]. To transmit a unit message within distance $d_{i j}$ from node $i$ to node $j$, the energy expending formula is shown as follows:

$$
E_{T x}\left(d_{i j}\right)=E_{\text {Telec }}+\varepsilon_{f s} \cdot d_{i j}^{\alpha}
$$

and to receive a unit message, the energy expending formula can be defined as:

$$
E_{R x}=E_{\text {Relec }}
$$

Therefore, the total energy consumption is illustrated by:

$$
\begin{aligned}
E_{i} & =E_{T x}\left(d_{i j}\right)+E_{R x} \\
& =\left\{\begin{array}{cc}
E_{\text {Telec }}+E_{\text {Relec }}+\varepsilon_{f s} \cdot d_{i j}^{2} & d_{i j}<d_{0} \\
E_{\text {Telec }}+E_{\text {Relec }}+\varepsilon_{\text {amp }} \cdot d_{i j}^{4} & d_{i j} \geq d_{0}
\end{array}\right.
\end{aligned}
$$

where $E_{\text {Telec }}$ is the transmitter electronics energy, $\varepsilon_{f s}$ and $\varepsilon_{\text {amp }}$ are coefficients of the free space model and multi-path model, respectively. $E_{\text {Relec }}$ denotes the receiver electronics energy, $\alpha$ indicates channel characteristics and is known as 2 or $4, d_{0}$ is a distance constant.

According to above formulas and the definition of cluster in [22], the network model could be constructed in Fig. 2, where the interval between $l_{1}$ and $l_{2}$ is called a Ring and the distance between two Rings is one-hop according to the definition of gradient in [20]. Therefore, the clusters could be formed between two circular orbits, which MEs move along for data collection.

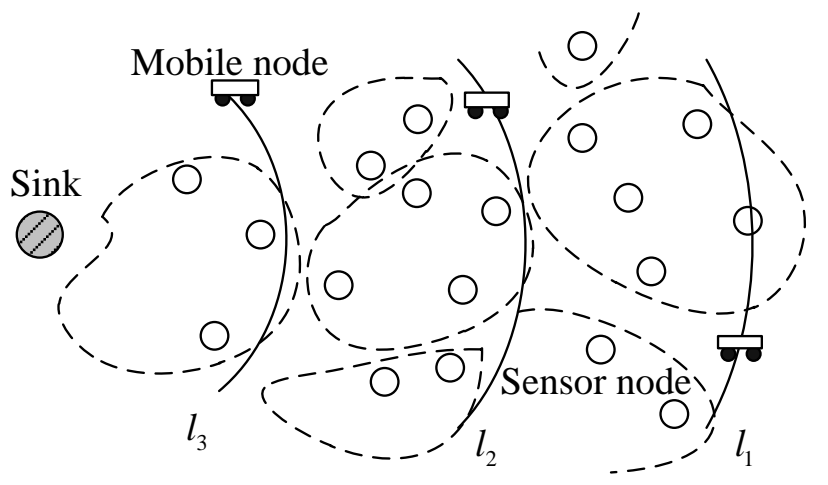

Fig. 2. Network Model with Mobile Elements.

In addition, the phenomenon of imbalanced energy consumption occurs due to the different transmitting distances from members to ME according to Equation (3). For convenience, we classfy members of a cluster into three groups, Range-Long nodes, Range-Short nodes and Rendezvous nodes. All types of nodes in the cluster are shown in Fig. 3, and they will be used to research the issue of energy consumption for data collection in the following sections.

Definition 1 (Range-Long nodes). Nodes marked by white circle in Fig. $\mathbf{3}$ are called Range-Long nodes nodes, if they satisfies

$$
S^{R L}=\left\{i \mid E_{i} \propto d_{i}^{4}\right\}
$$




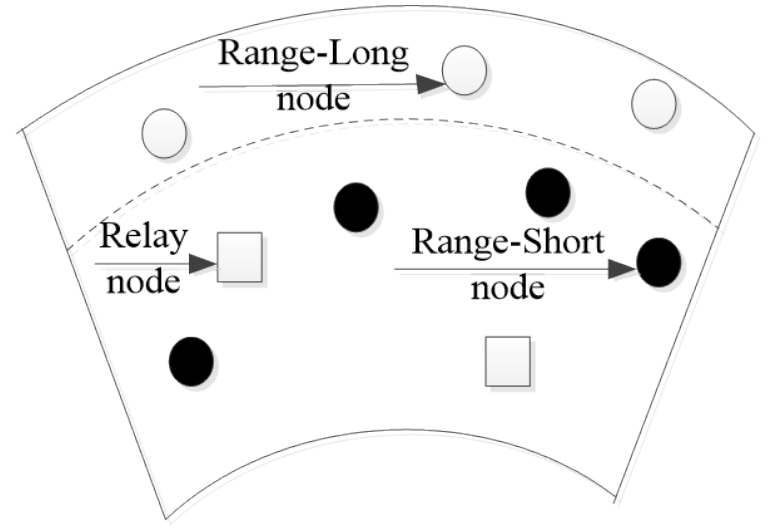

Fig. 3. Classification of Nodes in the Cluster.

Definition 2 (Rendezvous nodes). Rendezvous nodes (RNs) are defined as Relay nodes marked by white square in Fig. 3.

$$
S^{R}=\left\{i \mid i \in S_{R N}\right\}
$$

where $S_{R N}=\left\{R N_{1}, R N_{2}, \cdots R N_{m}\right\} \subset S, m$ is defined as the number of RNs in the cluster, $S$ indicates the set including all nodes of the cluster. Seen from the definition of set $S^{R L}$, the energy consumption of a node can be lowered through a relay node named RN. For convenience, we generally combine nodes of set $S^{R L}$ and set $S^{R}$ together and get a new set including Energy-saving nodes as follows.

\section{Definition 3 (Energy-saving nodes).}

$$
S^{E}=\left\{i \mid E_{i} \propto d_{i}^{4}\right\} \bigcup\left\{i \mid i \in S_{R N}\right\}
$$

Definition 4 (Range-Short nodes). Nodes identified by black circle in Fig. 3 are Range-Short nodes nodes, if they satisfies

$$
S^{R S}=S-S^{E}
$$

In this paper, we assume WSNs have the following two properties:

Assumption 1 The energy consumption of the node is dominated by Equation (3).

Assumption 2 Clusters in WSNs have been constructed.

In our network model in Fig. 2, the energy consumption of the node increases exponentially with the increase of transmission distance. Taking nodes between $l_{1}$ and $l_{2}$ in Fig. 2 for example, nodes close to $l_{1}$ consume more energy than nodes close to $l_{2}$ while ME moves on trajectory $l_{2}$ to collect data. In order to deal with this imbalanced energy consumption, we first introduce a RP for the cluster. In this case, we consider two aspects which include the strategy of one-hop modified clustering and the mobility of MEs for the energy efficiency.

\subsection{Mobility Based on Rendezvous Points}

We employ MEs moving along limited trajectories and determine RP for the cluster to achieve the optimization of energy consumption for data collection. And then, a series of problems should be solved in terms of the optimization on energy efficiency in the relative cluster: 1) whether an optimal RP exists on corresponding trajectory or not? 2) where is the RP located, and 3) how to determine an optimal RP if it exists. In order to solve above problems 
and improve the energy-efficient performance of WSNs, we propose a modified clustering scheme shown in Fig. 4. We assume that the networks have been clustered like what between $l_{1}$ and $l_{2}$ in Fig. 4 . It is easy to map every cluster into an arc on the trajectory curve $l_{2}$. For example, cluster 1 and cluster 2 in Fig. 4 are mapped into arc $\overparen{A C}$ and arc $\overparen{B C}$, respectively. Now, our goal is to design RP in each arc to make the energy consumption of the cluster least. We will demonstrate the procedure in detail next.

According to the RP shown in Fig. 4 and energy consumption model in (3), the workable solution to save energy should be utilized by reducing the transmission distance between nodes. Combined participation of MEs, the RP is introduced to guarantee the effect of energy saving. Therefore, the existence of RP must be proved firstly for data collection in terms of mobility.

Lemma 1. For a cluster, there exists one point named RP on a trajectory to make the energy consumption least when ME arrives at RP to achieve data collection, if $\alpha=2$.

Proof. We employ an approximate method to prove Lemma 1. Suppose that the minimality of energy consumption is to be solved in cluster 1 of Fig. 4. We divide $\overparen{A C}$ into $\mathrm{N}$ equally spaced sub-arcs $\left\{\widehat{A M_{1}}, \widehat{M_{2} M_{3}}, \cdots \widehat{M_{N-1} C}\right\}$, where the point set $\left\{A, M_{1}, \cdots, M_{N-1}, B\right\}$ is created naturally. Distinctly, when $\mathrm{N}$ is limited to infinity, one point $P_{i} \in\left\{A, M_{1}, \cdots, M_{N-1}, B\right\}$ is found to meet the following expression:

$$
\arg \min _{i}\left(\sum_{j=1}^{|s|} d_{i j}^{2}\right)
$$

where $j \in S$, and $d_{i j}$ denotes the distance between point $i$ and node $j$. According to the Equation (3), the expression (8) is proportional to $\arg \min _{i}\left(E_{i}\right)$. Because the item $d_{i j}^{2}$ in expression (8) is a constant for point $P_{i} \in\left\{A, M_{1}, \cdots, M_{N-1}, B\right\}$, it is easy to find the RP.

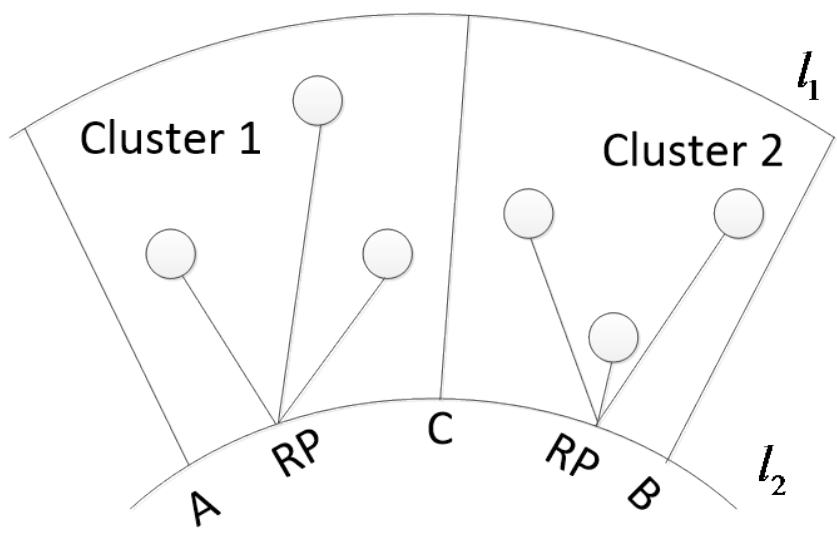

Fig. 4. Clustering Scheme.

\subsection{Rendezvous Node Selection Principle}

For further reduction of energy consumption, RNs could be selected to store and transmit data when ME arrives at RP. In the following section, the selection mechanism of RNs with energy consumption function is described in detail. 
Theorem 1. There exists a point $Q$ so that the direct communication model (3) between Rang-Long node and ME can be replaced by adding a relay sensor node at some point $\mathrm{Q}$ to reduce energy consumption, when $\alpha=4$.

Proof. Suppose that there exists the point Q (a RN in Fig. 5(a)) such that the following inequalities $d_{\text {node }, R N}<d_{0}, d_{R N, P N}<d_{0}$ and $d_{\text {node }, R P}>d_{0}$ are met. For convenience, let $d_{1}, d_{2}$ and $d_{3}$ illustrate $d_{\text {node,RN }}, d_{R N, P N}$ and $d_{\text {node }, R P}$, respectively. $E_{1}$ is the energy consumption between node and RN(i.e., Q ). $E_{2}$ indicates energy consumption of RN, and $E_{3}$ denotes energy consumption between Rang-Long node and RP. They are concretely calculated as follows.

$$
\begin{gathered}
E_{1}=E_{\text {Telec }}+\varepsilon_{f s} \cdot d_{1}^{2} \\
E_{2}=E_{\text {Relec }}+E_{\text {Telec }}+\varepsilon_{f s} \cdot d_{2}^{2} \\
E_{3}=E_{\text {Telec }}+\varepsilon_{m p} \cdot d_{3}^{4}
\end{gathered}
$$

We will verify that whether the sum of $E_{1}$ and $E_{2}$ is less than $E_{3}$ or not. Therefore, we combine $E_{1}$ and $E_{2}$ to subtract $E_{3}$ :

$$
\begin{aligned}
\Delta E & =E_{1}+E_{2}-E_{3} \\
& =\varepsilon_{f s} \cdot\left(d_{1}^{2}+d_{2}^{2}\right)+\left(E_{\text {Relec }}+E_{\text {Telec }}\right)-\varepsilon_{\text {amp }} d_{3}^{4}
\end{aligned}
$$

Due to $d_{1}, d_{2} \in\left(0, d_{0}\right], d_{3} \in\left(d_{0},+\infty\right)$, Equation (12) can be rewritten

$$
\begin{aligned}
\Delta E & =E_{1}+E_{2}-E_{3} \\
& =\varepsilon_{f s} \cdot\left(d_{1}^{2}+d_{2}^{2}\right)+\left(E_{\text {Relec }}+E_{\text {Telec }}\right)-\varepsilon_{\text {amp }} d_{3}^{4} \\
& \leq 2 \varepsilon_{f s} \cdot d_{0}^{2}+\left(E_{\text {Relec }}+E_{\text {Telec }}\right)-\varepsilon_{\text {amp }} d_{3}^{4}
\end{aligned}
$$

Because the values of $\varepsilon_{f s}, \varepsilon_{\text {amp }}, d_{0}$, and $E_{\text {Relec }}+E_{\text {Telec }}$ in (13) are all positive constants, there exists a $d_{3} \in\left(d_{0},+\infty\right)$ to make $\Delta E<0$ worable. So we prove the existence of the RN.

Remark 1. When $\alpha=2$, it is not necessary for RN to relay data. The reason is that the distance between the node and ME is short, which the variation of energy expenditure for every node is very little. In fact, we deal with data transmission directly from nodes to ME if all the transmission distances are less than $d_{0}$ in the model (3).

Remark 2. When $\alpha=4$, this illustrates that long distance transmission leads to enormous energy consumption. One effective way to reduce the energy is to shorten the transmission range by multi-hop relays.

In order to further reduce energy consumption, we exploit RNs to relay data. However, choosing appropriate quantities of RNs is difficult as far as the optimization of energy efficiency is concerned.

Theorem 2. It is a NP-hard problem to select RNs for energy efficiency in the cluster.

Proof. We will reduce the problem of RN selection into a knapsack problem. Suppose that $E_{3}$ stands for the energy consumption shown in Theorem 1 . We employ a RN as a relay node to lower energy consumption of the cluster, since energy consumption can exponentially increase with increasing distance. $E_{1}$ and $E_{2}$ are energy consumption related to the relay node 
RN. So $\Delta E=E_{1}+E_{2}-E_{3}$ can be regarded as a cost of RN. For the selection of relay node $\mathrm{RN}$, both $E_{1}+E_{2}$ and $\Delta E$ can be mapped into the weight and price of items in knapsack problem, respectively. If we designate an energy consumption bound $\Psi$ for the cluster as the total constrain of weight in knapsack problem, we can select $E_{1}+E_{2}$ (i.e. RN) to maximize the expression $\left|\sum_{i=1}^{N} \Delta E\right|$. Then we succeed to build one-to-one map between RN selection and knapsack problem based on the above specifications. In the end, the expression max $\left|\sum_{i=1}^{N} \Delta E\right|$ is mapped into the maximal price of items in the knapsack. Therefore, $\mathrm{RN}$ selection problem is reduced into the knapsack problem which is well-known as a NP-hard. Hence, RN selection problem is also NP-hard.

In order to achieve energy efficiency in our data collection framework, we give the following theorem.

Theorem 3. In the cluster, there exist the RP with ME to collect data and RNs as relay nodes to ensure that the energy consumption is least when $\alpha=2$ and $\alpha=4$ coexist.

Proof. In our data collection framework, we can reduce energy consumption according to theorem 1 when $\alpha=4$ is workable. In this way, only partial one-hop nodes including nodes nearby RP and RNs can communicate with ME, which is a special example of lemma 1 . So we conclude it.

\section{Rendezvous Node Selection Strategies for Energy Efficiency}

In order to make a clarification for energy-efficient data collection, we design a triple tuple $<$ Node, RN,RP $>$ to represent the data transmission route sequence, where the three elements indicate Range-Long node, Relay node and data transmission terminal ME, respectively. Especially, RN in the triple tuple < Node, RN,RP> may be NULL, i.e. $<$ Node, NULL, RP $>$ or $<$ Node,RP $>$. In the scenario, the node actually sends data to ME directly when ME arrives at RP.

\subsection{Transmission Model of Rendezvous Node Selection}

In terms of energy efficiency for data collection, RN plays a rendezvous role to relay data from Range-Long nodes. Determining RN in the cluster always incurs the dilemma which node should be selected as RN, so we analyze loacation states of RN and summarize four scenarios to deal with the energy balance issue according to transmission model. In the process, we assume that the mission of RN is just for data relay in terms of saving and balancing energy expenditure. Definitely, we expound the four scenarios about RN in detail, which affect the strategies for $\mathrm{RN}$ selection.

Case 1: General rendezvous case. As show in Fig. 5(a), RN close to RP is chosen as a relay node to reduce energy expenditure.

Case 2: Parallel rendezvous case. The energy consumptionn of route $<$ Node, RN1,RP $>$ may be equal to the one of $<$ Node, RN2,RP $>$ due to the structure in Fig. 5(b).

Case 3: Symmetry rendezvous case. The energy consumptionn of route $<$ Node, RN1,RP $>$ may be equal to the one of $<$ Node, RN2,RP $>$ as shown in Fig. 5(c). 
Case 4: Sharing rendezvous case. Node1 and Node2 share RN as a relay node as shown in Fig. 5(d).

Note that, RN1or RN2 in Case 2 and Case 3 is selcted in a random manner to reduce energy consumption. In Case 4, Node1 or Node2 will be chosen to transmit data via RN.

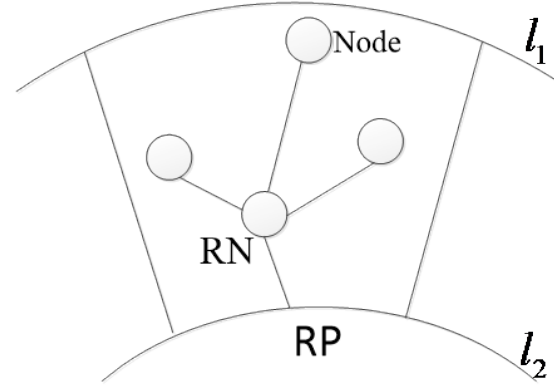

(a)

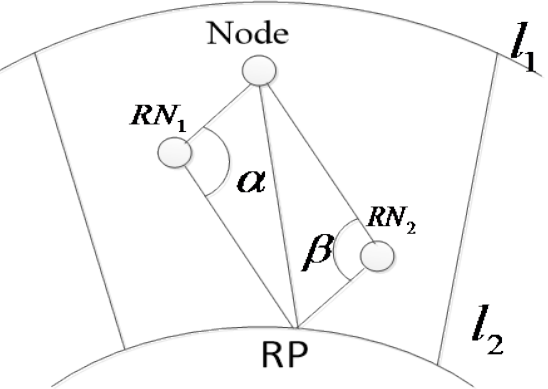

(b)

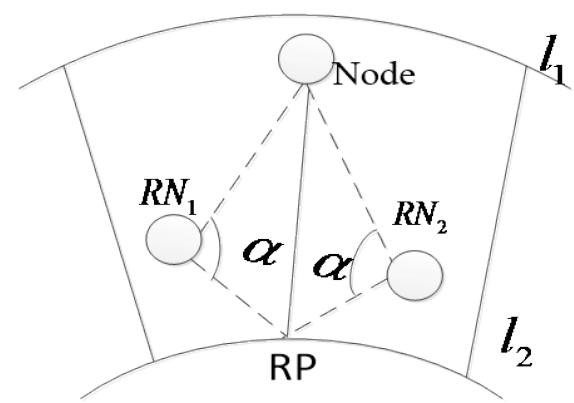

(c)

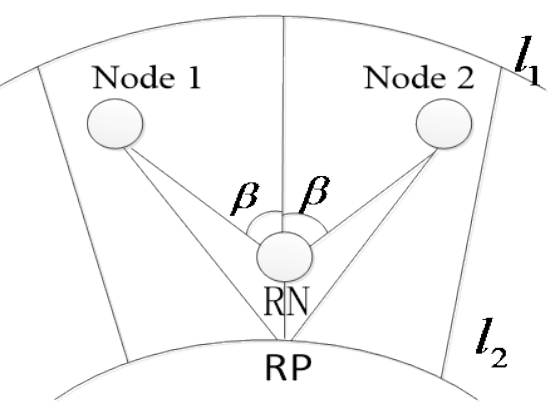

(d)

Fig. 5. Scenarios of a RN Selection

\subsection{Application Principle of Diminishing Marginal Utility Rule}

Subsection 4.1 demonstrates the complexity of RN selection. To deal with it and achieve energy optimization, we introduce an economic theory named DMUR, which is employed in the process of data collecting and needs be indispensable to reveal its evolution mechanisms. For convenience, we assume that the energy optimization is studied in the cluster S. According to Equation (3), the optimization model of energy consumption for unit data size can be built

$$
\begin{aligned}
& \min \sum_{\mathrm{i}=1}^{|S|}\left(\varepsilon_{f_{s}} \cdot d_{\bar{i}}^{\alpha}+E_{\text {Telec }}\right) \\
& \text { s.t. } \frac{1}{|S|} \sqrt{\left(E_{1}^{S}-\bar{E}\right)^{2}+\left(E_{2}^{S}-\bar{E}\right)^{2}+\cdots+\left(E_{|S|}^{S}-\bar{E}\right)^{2}} \leq \Phi
\end{aligned}
$$

where $|S|$ is the size of $S, E_{i}^{S}$ denotes residual energy of node $i, \bar{E}$ indicates average residual energy, $\boldsymbol{d}_{i}$ stands for data transmission range. Generally, $\Phi$ indicates an empirical value with preassigned threshold in practice and is hard to manually operate for complicated scenarios. In order to solve this problems encountered in the constrained optimization (14), we utilize RNs as relay nodes and ME-assisted RP for energy efficiency. Before proposing our solution, we introduce DMUR based on a utility function about energy consumption. Below is the definition of the utility function according to Equation (12): 
Definition 5 (Utility function). A utility function about energy is presented by

$$
f_{t}(n)=\sum_{i=1}^{n}\left(E_{i 1}^{t}+E_{i 2}^{t}-E_{i 3}^{t}\right)
$$

where $t$ indicates time and $n$ is the number of RNs in the cluster $S$ with the assumption of $n \geq 1, E_{i 1}, E_{i 2}$ and $E_{i 3}$ are calculated from expression(9)-(11).

In Equation (15), we can exactly search a set of $\left\{R N_{1}, R N_{2} \cdots, R N_{n}\right\}$ by calculating the value $f_{t}(n)$, so that it is easy to satisfy the following inequality

$$
f_{t}(n+1)+f_{t}(n-1)<2 f_{t}(n)
$$

And then we propose a quantitative analysis method to search RNs and get the optimal number of them in a cluster.

Theorem 4. The number of RNs $n$ is $\arg \min _{n}\left(f_{t}(n+1)-f_{t}(n)\right)$, if a utility function about energy consumption is defined as $f_{t}(n)=\sum_{i=1}^{n}\left(E_{i 1}^{t}+E_{i 2}^{t}-E_{i 3}^{t}\right)$.

Proof. Obviously, the utility function $f_{t}(\cdot)$ is just for $\alpha=4$, which is a monotonically decreasing function with increment of $n$. Next, we look for RNs in the cluster according to Inequality (16). Actually, Inequality (16) is a DMUR in terms of utility function $f_{t}(n)$. With $n$ increasing constantly, the following equality is satisfied mathematically.

$$
\lim _{n \rightarrow+\infty} f_{t}(n+1)-f_{t}(n)=0
$$

In reality, the number of RNs is very finite in a cluster. Generally, as long as we guarantee the marginal increment between $f_{t}(n+1)$ and $f_{t}(n)$ is minimum, that is,

$$
\min \left(f_{t}(n+1)-f_{t}(n)\right)
$$

the number of RNs can be illustrated

$$
\arg \min _{n}\left(f_{t}(n+1)-f_{t}(n)\right)
$$

\subsection{Adaptive Energy Consumption Optimization}

To achieve energy optimization and energy balance, we improve the constrained optimization (14) according to Theorem 3 and Definition 5. After searching a set of RNs, we can determine the energy-efficient optimization with MEs.

$$
\begin{aligned}
& \min \left(\sum_{i=1}^{\left|S^{R L}\right|}\left(\varepsilon_{f s} \cdot d_{i, R N}^{2}\right)+\sum_{j=1}^{\left|S-S^{R L}\right|}\left(\varepsilon_{f s} \cdot d_{j, R P}^{2}\right)\right)+C \\
& \text { s.t. } f_{t}(n+1)+f_{t}(n-1)<2 f_{t}(n) \\
& \operatorname{var}_{n+1}\left(E_{1}^{S}, E_{2}^{S}, \cdots E_{|S|}^{S}\right)<\operatorname{var}_{n}\left(E_{1}^{S}, E_{2}^{S}, \cdots E_{|S|}^{S}\right) \\
& d_{i, R N}<d_{0} \\
& d_{j, R P}<d_{0}
\end{aligned}
$$


where $\operatorname{var}(\cdot)$ indicates variance and $C$ denotes an energy constant calculated according to Equation(3)

$$
C=\left|S^{R L}\right| \cdot E_{T x}+\left|S-S^{R L}\right| \cdot\left(E_{T x}+E_{R x}\right)
$$

Note that the proposed DMUR can be used for selecting optimal combination of RNs while guaranteeing the balance of energy consumption with energy variance in the cluster.

After that, ME is used to receive data from the cluster. Due to the latency causeded by a single ME, we exploit multiple MEs to collect data with RN selection mechanism for energy efficiency in Algorithm 1 named RTA.

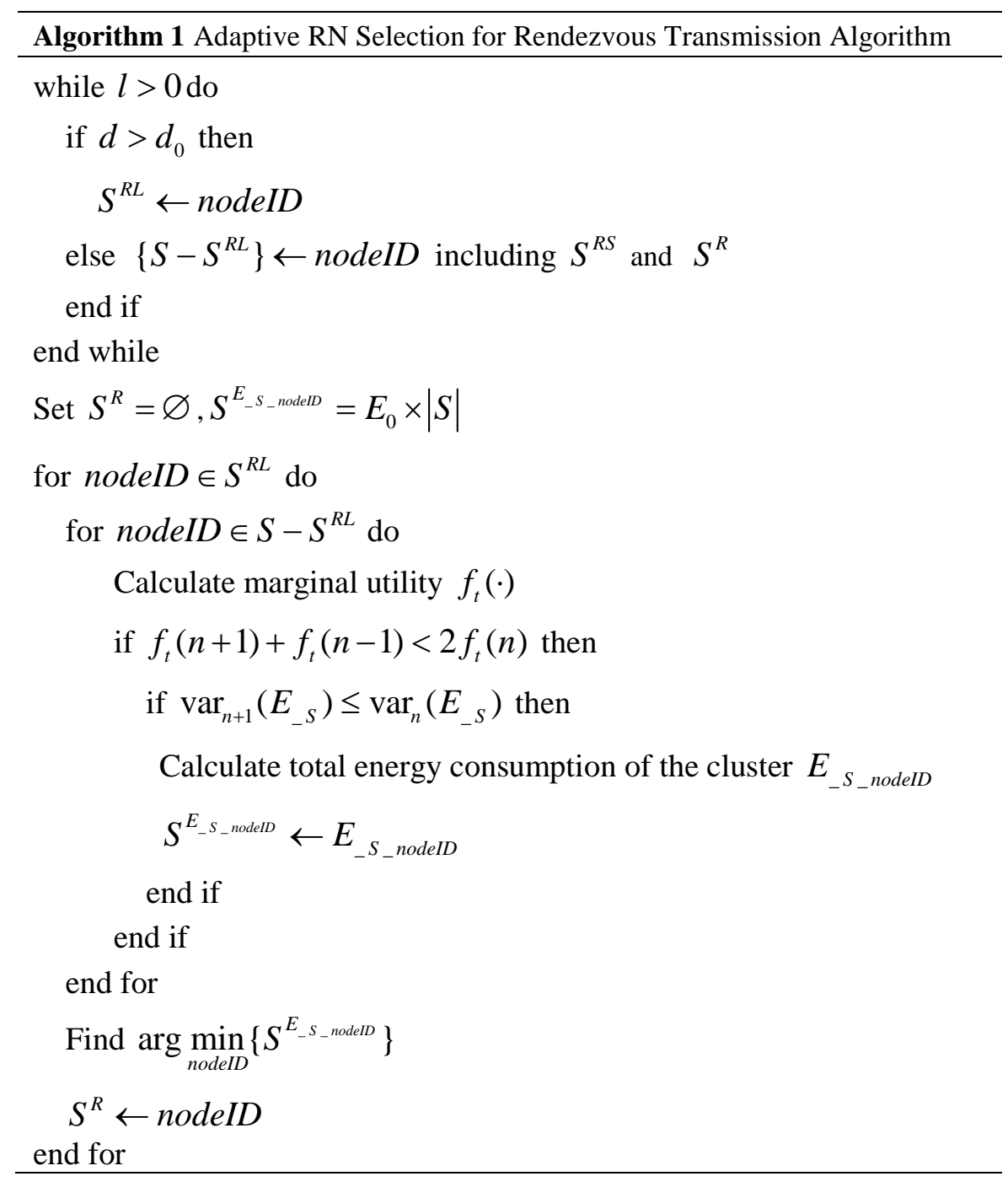

In Algorithm $1, E_{0}$ is the initial energy of every node, $E_{-} S$ is the total energy of set $S$, and

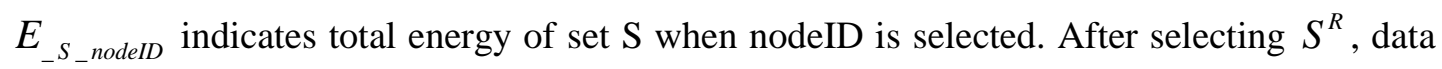
are stored at corresponding RNs until the ME arrives at the RP. Since the position of every node is known for other node in the cluster, it is easy for every node to select the RN in terms 
of energy efficiency. We briefly describe the scheme of data transmission after RN being selected in Fig. 5(a).

\section{Improvement for Energy Efficiency Optimization}

In this section, we first analyze the cooperation between MEs, and then demostrate the specific improvement strategies for energy- efficient optimization.

\subsection{Mobile Element Cooperation}

Comparing with the single ME strategy, we allocate several MEs shown in Fig. 2. Suppose that ME travels with a speed $\boldsymbol{v}$ and arrives at RP at time $T$. Like the definition of deadline for data transmission, we also calculate a deadline $\Delta t=|T-t|$. Generally, we assign the deadline as follows:

$$
\Delta t=\max |T-t|
$$

where $\max (\cdot)$ ensures enough MEs on a trajectory. Then we can calculate the number of MEs

$$
\operatorname{Num}_{\bar{i}}=\left\lceil\frac{2 \pi R_{\bar{i}}}{\Delta t \times v}\right\rceil
$$

where $R_{i}$ denotes the radius of trajectory $l_{i}$ shown in Fig. 2 . The symbol $\lceil\cdot\rceil$ indicates an upper bound. So, we can calculate the number of MEs on $l_{i+1}$.

$$
N u m_{i+1}=N u m+\left\lceil\frac{2 \pi\left(R_{i+1}-R_{i}\right)}{\Delta t \times v}\right\rceil
$$

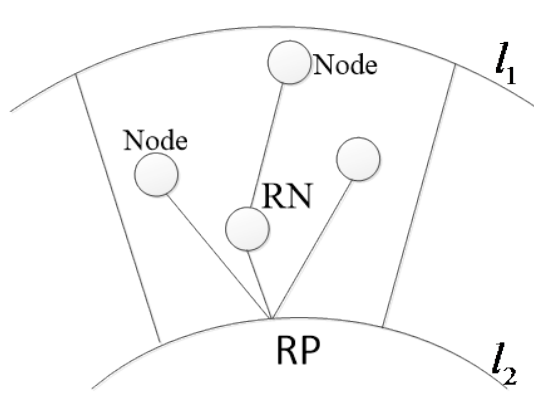

(a)

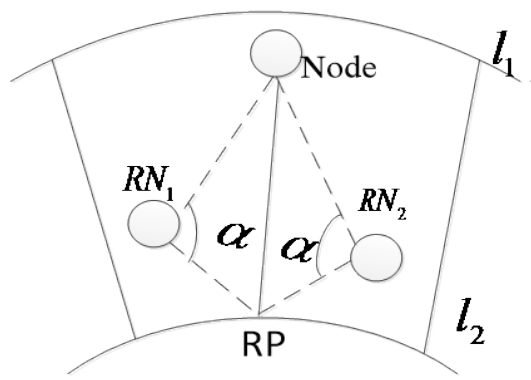

(c)

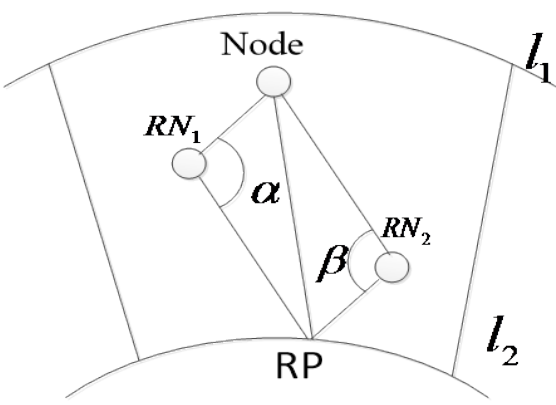

(b)

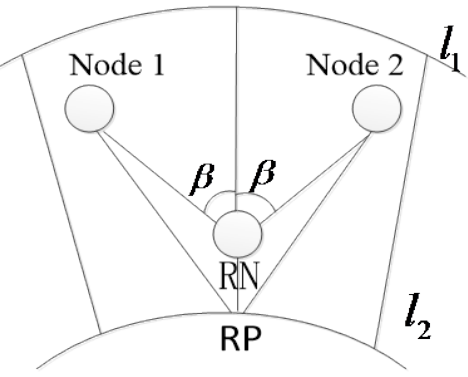

(d)

Fig. 6. Algorithm of RN Selection in Step 1 


\subsection{Two-Orbit Back-Propagation Algorithm}

In the network framework, we proposed TOBPA for a lower delay to improve the efficiency of data collection. As shown in this algorithm bellow, we design two steps to reduce the time interval of data collecting so that the problem of delay can be weakened.

Sept 1) RNs relay. We select RNs to relay data from Range-Long nodes to RP in terms of optimizing energy consumption of Range-Long nodes and weakening energy unbalance. Different from algorithm 1, Range-Short nodes in TOBPA will communicate with ME like in Fig. 6(a), other scenarios are the same as Fig. 5(b-d).

Step 2) Reversed Data Transmission. In order to improve the efficiency of data collection and lower the delay in step 1 , we create a reversed data transmission mechanism called TOBPA, which have the similar concepts about RNs and reversed rendezvous points (R-RPs) in terms of what in step 1.

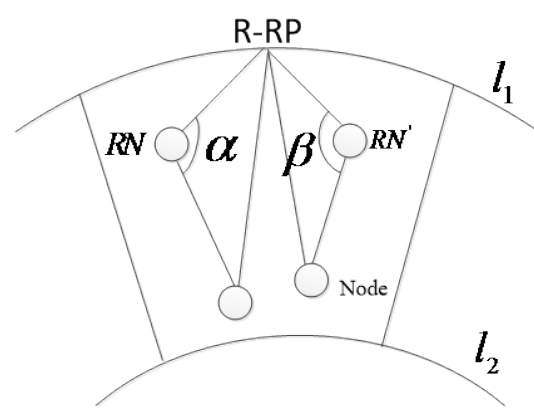

(a)

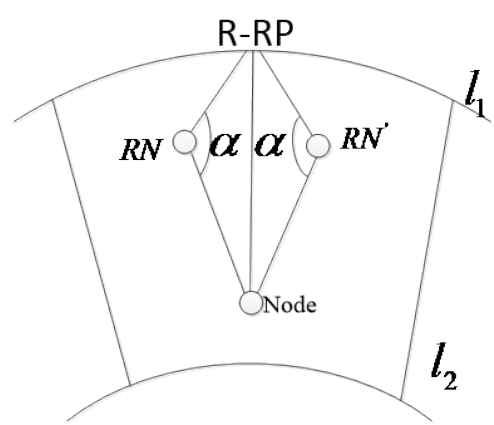

(c)

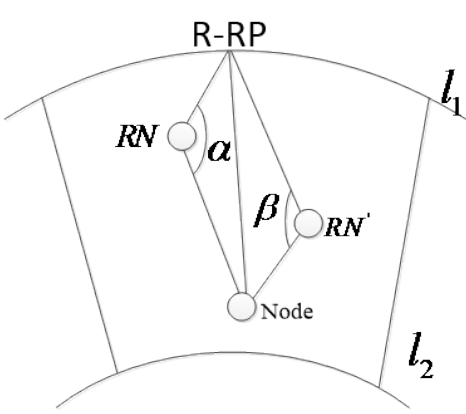

(b)

Fig. 7. Algorithm of RN Selection in Step 2

As shown in Fig. 7, the R-RP also plays the similar role as the RP for ME. Differently, Range-Long node close to $l_{1}$ in step 1 alters the role as RN or Range-Short node, while partial Range-Short nodes close to $l_{2}$ in step 1 become Range-Long nodes instead, others change their roles in step 2 according to DMUR. Anyhow, similar conlusions inTOBPA could be acquired in parallel. Therefore, we naturally come to the following corollaries without proof processes.

Corollary 1. In the reversed data transmission process, there exists one point named R-RP on the trajectory to make energy consumption of cluster is least when ME arrives at R-RP to achieve data collection, if $\alpha=2$. 
Corollary 2. In the reversed data transmission process, there exists a point $\mathrm{Q}$ so that the direct communication model (3) between Range-Long node and ME can be replaced by adding a relay sensor node at point some $\mathrm{Q}$ to reduce energy consumption when $\alpha=4$.

Corollary 3. In the reversed data transmission process, it is a NP-hard problem to select RNs for energy efficiency in the cluster of Fig. 7.

Corollary 4. In the reversed data transmission process, there exist the R-RP with ME to collect data and RNs as relay nodes to ensure that the energy consumption of cluster is least when $\alpha=2$ and $\alpha=4$ coexist in the cluster.

Corollary 5. In the reversed data transmission process, the number of R-RNs $N$ is $\arg \min _{N}\left(f_{T}(N+1)-f_{T}(N)\right)$, if a utility function about energy consumption is defined as follows: $f_{T}(N)=\sum_{i=1}^{N}\left(E_{i 1}^{T}+E_{i 2}^{T}-E_{i 3}^{T}\right)$.

Note that except for reducing energy expenditure by shortening the transmitting distance, what involved in TOBPA is the decrease of data volume, which not only reduces energy consumption explicitly but also adjusts energy balance implicitly. We will concretely elaborate the process next.

\subsection{Strategy design on Improvement of Energy efficiency}

We analyze the energy optimization and energy balance when ME arrives at RP or R-RP. Because energy consumption distributes differently for step 1 and step 2, Range-Short nodes in step 1 could be reused inevitably when ME stops at the R-RP to collect data in step 2. On the other hand, many MEs could stop at RP or R-RP frequently in a time interval according to network structure Fig. 2, Fig. 6 and Fig. 7, which leads to a harmful energy dissipation for Range-Short nodes to transmit data. It is reasonable for Range-Short nodes to be reduced the utilized frequency, so we exploit a strategy to improve energy efficiency, which permit every Range-Short node to be scheduled once during the data collection cycle $[t, t+\Delta t]$. The strategy is detailedly shown in Fig. 8.

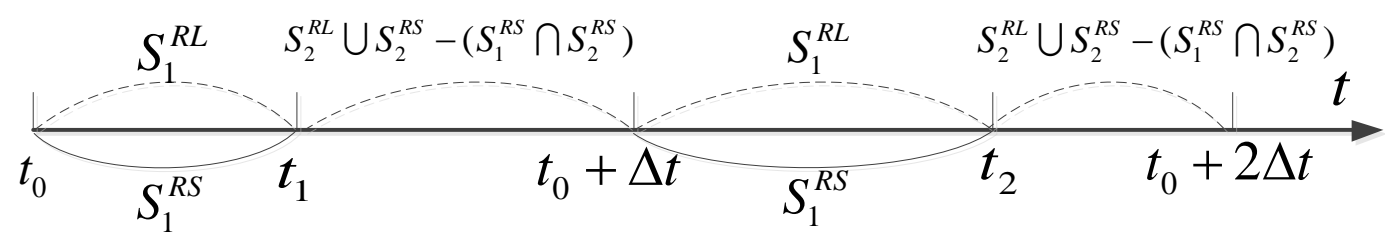

Fig. 8. Working States of Range-Long and Range-Short Nodes.

Seen from Fig. 8, no data are sensed and transmitted by Range-Short nodes during the time interval $\left[t_{1}, t_{0}+\Delta t\right]$ in terms of a data collection loop. Consequentially, less data need to be transmitted, which results in less energy to be consumed. Actually, this mechanism can guarantee that the data collected by different MEs from the same cluster at different time could be integrated at Sink without losing performances of WSNs. The data collection algorithms named TOBPA are listed in algorithm 2 and algorithm 3. 

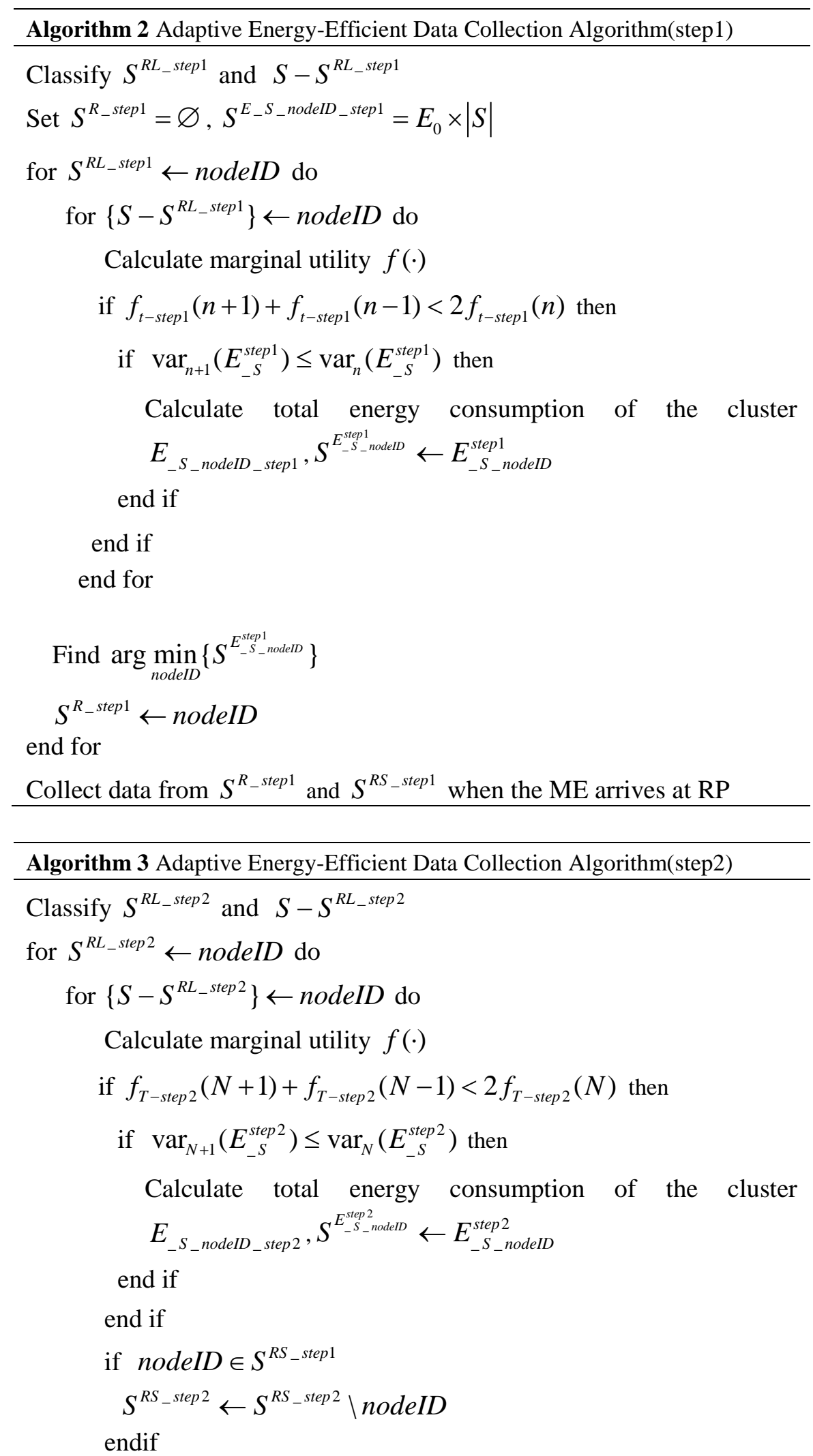


$$
\begin{aligned}
& \text { Find } \arg \min _{\text {nodelD }}\left\{S^{E_{-S_{-} \text {nodel }}^{\text {step }}}\right\} \\
& S^{R_{-} \text {step } 2} \leftarrow \text { nodeID }
\end{aligned}
$$

end for

Collect data from $S^{R_{-} \text {step2 }}$ and $S^{R S_{-} \text {step 2 }}$ when the ME arrives at R-RP

Note that: 1) The algorithm of sept 1 is different from algorithm 1 due to the different transmission destination of the Range-Short nodes; 2) In the process of step 2, although ME collects data from Range-Long nodes and partial Range-Short nodes, which may lead to a temporary energy imbalance, this will be amended by the RN selection in the next loop in terms of step 1.

\section{Simulation Results}

In the section, we carry out the simulations by MATLAB 2012b on a Lenovo Laptop with a 2.5 GHz Core4 Inter(R) Core(TM) i5-3210M CPU and 4 GB RAM. Suppose that nodes are randomly deployed in a region of interest and we first list parameters in Table 1 before we illustrate simulation results of our algorithms.

Table 1. Simulation parameters

\begin{tabular}{|c|c|c|c|}
\hline parameters & value & parameters & value \\
\hline \hline$\alpha$ & $2 / 4$ & $\varepsilon_{f s}$ & $10 \mathrm{~J} / \mathrm{bit} / \mathrm{m}^{2}$ \\
\hline$d$ & $90 \mathrm{~m}$ & $\varepsilon_{m p}$ & $0.0013 P \mathrm{~J} / \mathrm{bit} \mathrm{m}^{4}$ \\
\hline$E_{0}$ & $0.5 \mathrm{~J}$ & $n_{\text {num }}$ & 100 \\
\hline$E_{\text {elec }}$ & $50 \mathrm{~J} / \mathrm{bit}$ & Fixed Sink & $(0,0)$ \\
\hline
\end{tabular}

When the first node is dead, we compare our RTA and TOBPA with Cluster-based Algorithm which here is assume that data are collected by members and routed to Sink through CHs (e.g. LEACH [30]). Through scheduling the MEs in TOBPA, we can make the lifetime of three of them comparable.

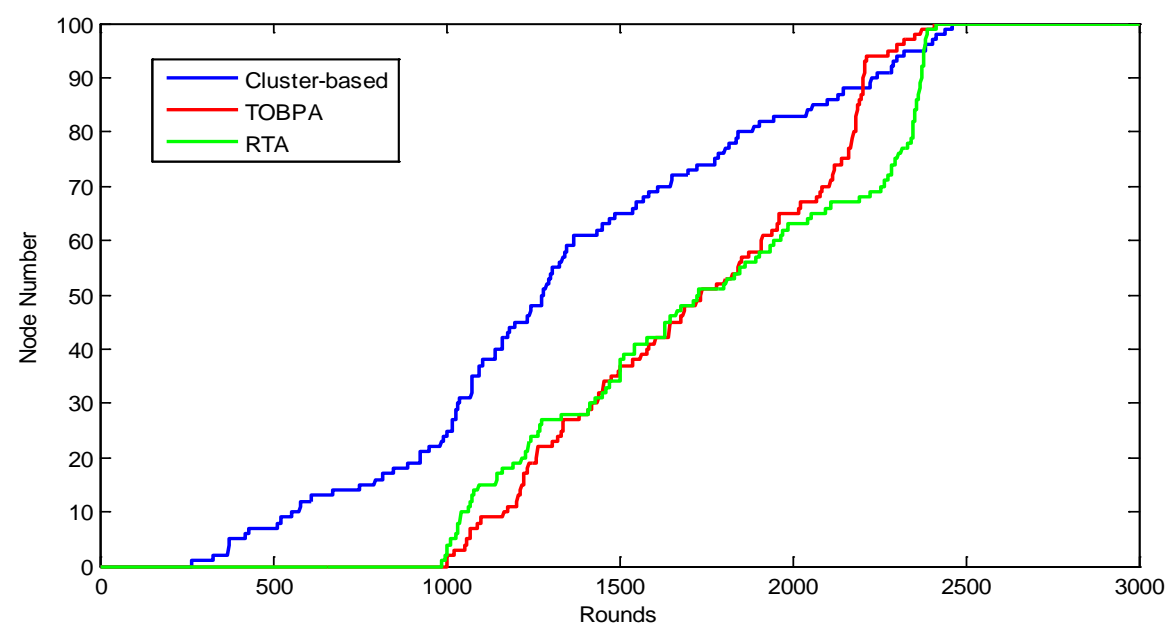

Fig. 9. Lifetime of Three Algorithms. 
In Fig. 9, long distance transmission leads to less than 500 rounds in terms of the lifetime of Cluster-based WSNs. For RTA, we introduce MEs and determine RNs as relay nodes to reduce the transmission distance. So the lifetime is longer that Cluster-based Algorithm. In this process, although RNs afford more energy consumption, DMUR guarantees the RNs selection mechanism dynamically adapts the tradeoff between minimum energy consumption and energy balance in the cluster. Compared with RTA, TOBPA has a little longer lifetime, because TOBPA activates only partial sensor nodes in step 2.
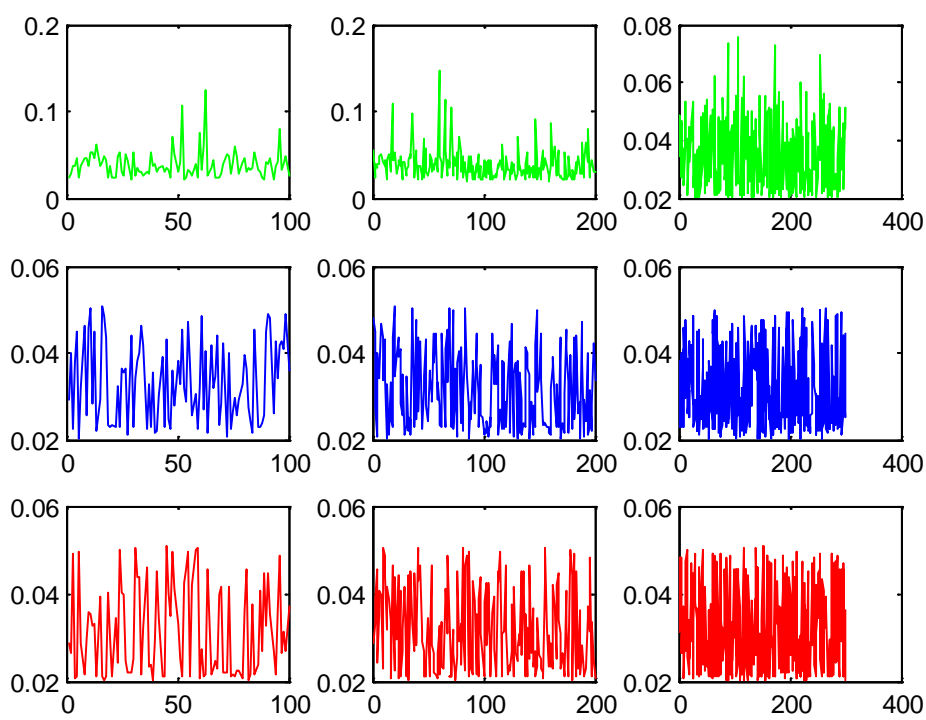

Fig. 10. Node Energy of Different Scales.

In order to evaluate the ubiquity for energy-efficient data collection, we design three scales of WSNs(i.e. 100nodes, 200nodes and 300nodes) and analyze the energy expenditure of these scenarios in 100 rounds. As shown in Fig. 10, the first row demonstrates energy consumption of Cluster-based algorithm, the second row is RTA's, and the third is TOBPA's. Cluster-based algorithm in the first row absolutely consums most energy due to the long transmission distances of nodes. For instance, node ID 52 and node ID 63 for the scale of 100 nodes, node ID 18,ID 60 and ID 65 for 200 nodes, and ID 88,ID 107, ID 172 and ID 256 for 300 nodes. For the second row, the value of energy expenditure just fluctuates from 0.02 to 0.05 for different scales. The reason is that RTA exploits RNs to relay data to save energy instead of directly transmitting data to ME while adaptively adjusting energy balance among nodes according to DMUR, which leads to an obviously longer lifetime than Cluster-based algorithm. Seen from the third row, OTBPA keeps effect as good as RTA. The reason is that TOBPA improves data transmission frequency to decrease the impact of delay problem without increasing more energy consumption of each node.

Then we evaluate the impact of different transmission radius ranged from $60 \mathrm{~m}$ to $120 \mathrm{~m}$. Seen from Fig. 11, the lifetime of Cluster-based algorithm declines with transmission distance growth. Especially, when the radius is $\mathrm{R}=60$, no $\mathrm{RN}$ is available so that three of them illustrate the same features. When the radius meets $\mathrm{R}=90$ or $\mathrm{R}=120$, RTA and TOBPA manifest obviously advantages compared with Cluster-based Algorithm. Due to the reversed transmission without increasing visit frequency, TOBPA can actually save a little more energy than RTA, which incurs more rounds than RTA. In addition, values of rounds for TOBPA and 
RTA are increasing with node scale growth owing to RN selection for energy efficiency.

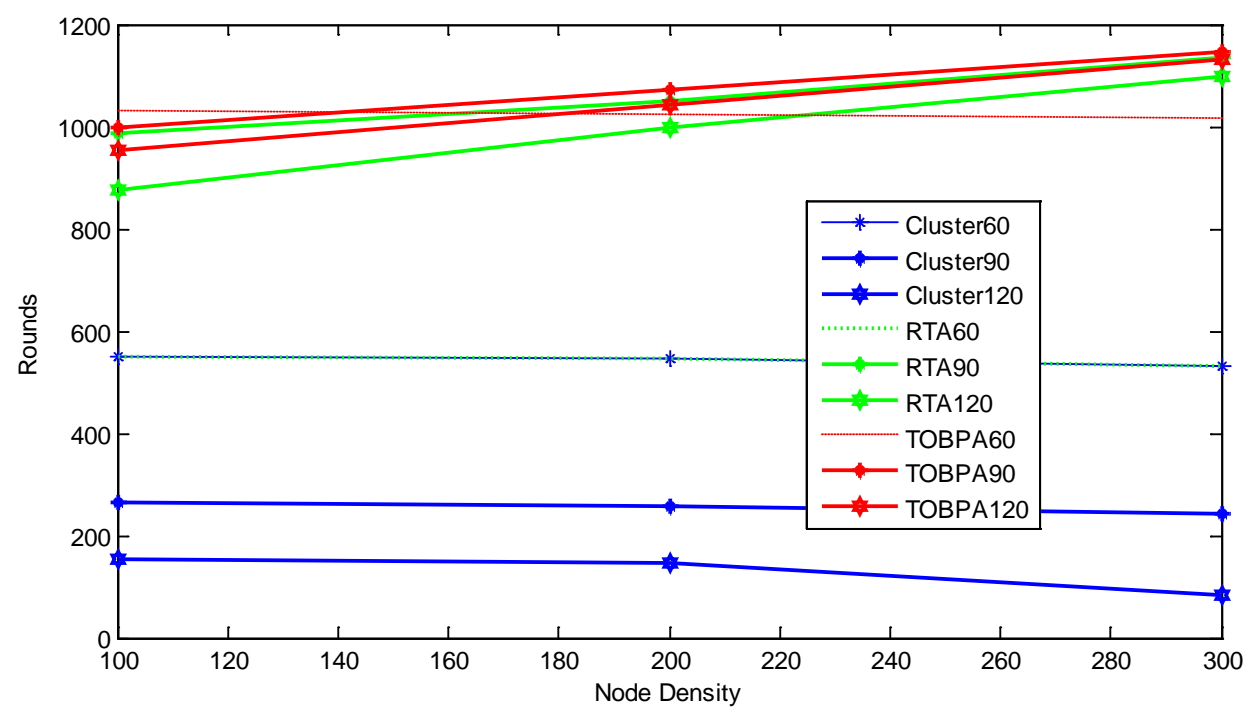

Fig. 11. Impact of Radius.

In order to impartially evaluate the performances of all algorithms, the criteria must be consistent. So we still employ a peculiar criterion (i.e. the same visits and total packages for a cluster, not the same time) to measure the issue of energy consumption or energy efficiency according to the rule in step 2 of TOBPA. The residual energy is explored when the first node is death with $\mathrm{R}=90$ in Fig. 12. Cluster-based mechanism is displayed in the upper left part; RTA in the upper right part; TOBPA in the bottom left part and their variances in the bottom right part.
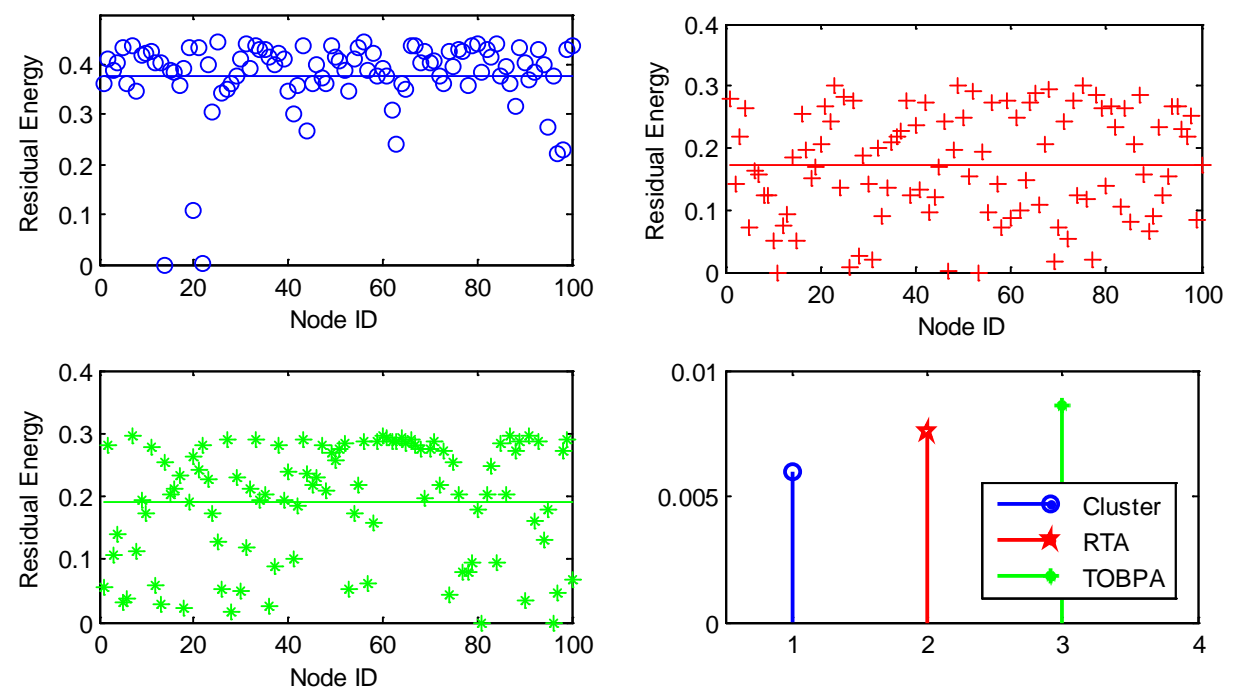

Fig. 12. Residual Energy and Variance.

From Fig. 12, Cluster-based Algorithm makes most nodes keep 80\% energy when the first node is dead, while RTA and TOBPA are almost $40 \%$. Unquestionably, DMUR dominates 
the RN selection strategy to deal with energy balance and energy optimization, which contributes to residual energy with a smaller mean in terms of RTA or TOBPA. Compared to RTA, TOBPA have a little bit higher mean due to the assumption of the same visits and total packages for data collection. In fact, the scheduling mechanism wakes up partial Range-Short nodes in step 2 of TOBPA but partly increases the load of them, which temporarily demostrates some negative effects in terms of energy consumption imbalance. Fortunately, this problem will be amended by selecting right RNs in next loop.Therefore, the residual energy variances of the three algorithms clearly illustrate this phenomenon.

In Fig. 13, we show the relationship between RNs and Range-Long nodes, which illustrates their quantities respectively. For different scales of network including 100, 200 and 300 nodes, Range-Long nodes are more than RNs. In fact, several Range-Long nodes may share one RN in RTA and TOBPA, which determine the RNs by DMUR from the perspective of energy optimization and energy balance.

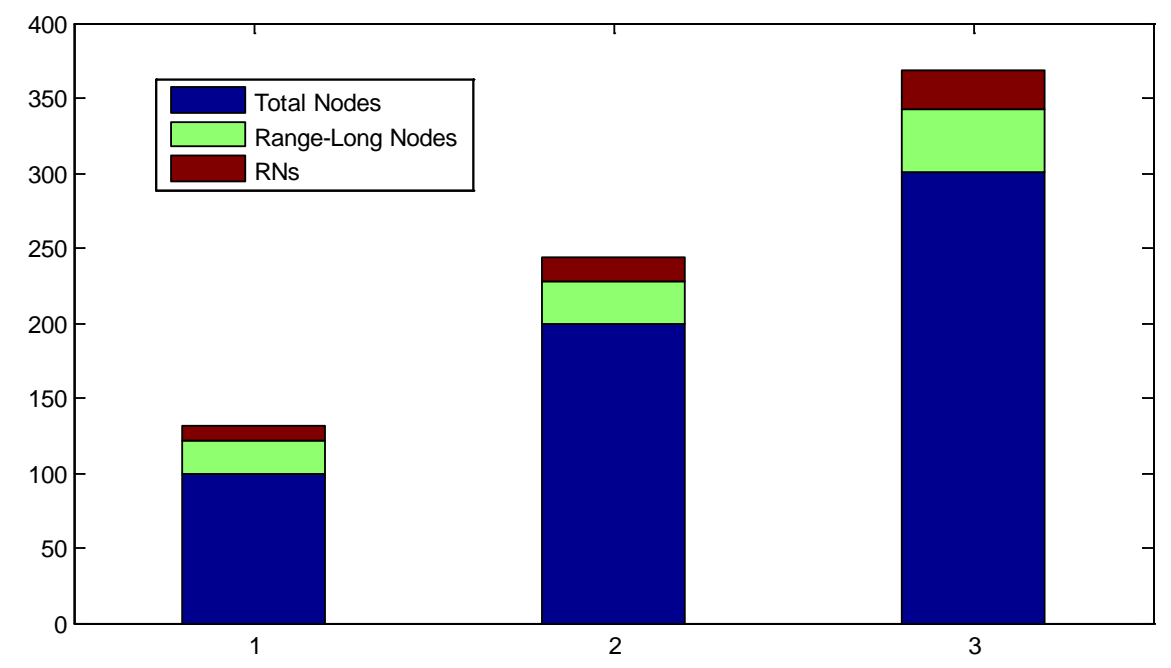

Fig. 13. The Number of RNs and Range-Long Nodes.

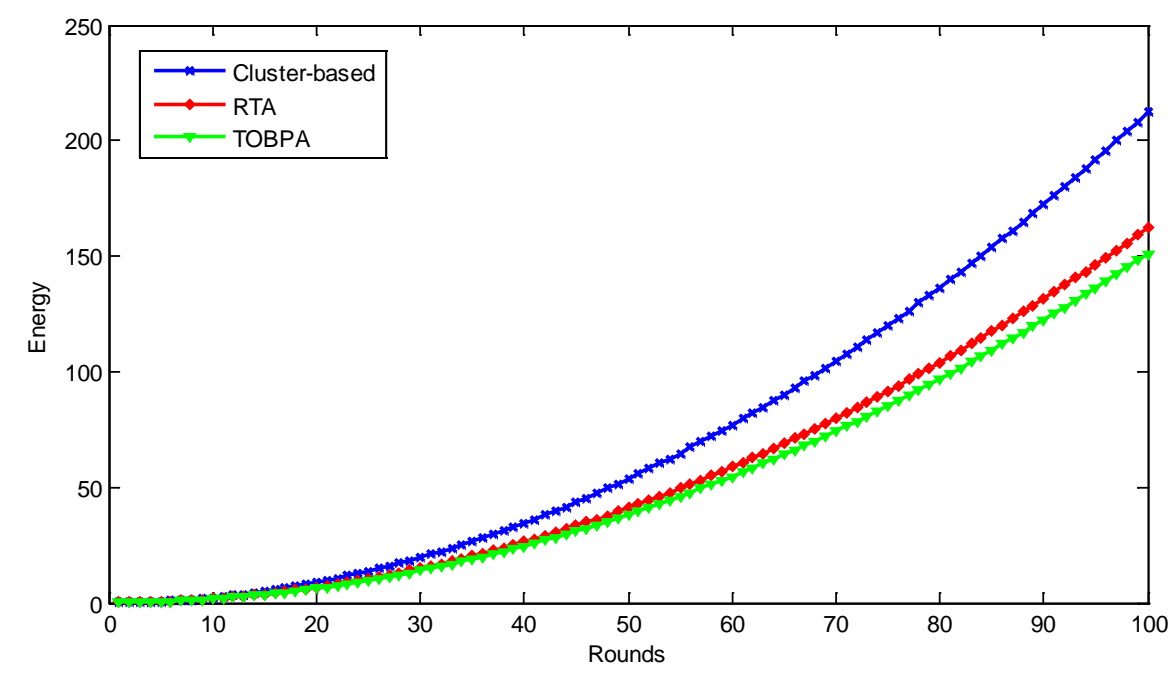

Fig. 14. Accumulated energy consumption. 
For comparability, we evaluate accumulated energy consumption with radius $\mathrm{R}=90 \mathrm{~m}$ in 100 rounds. In Fig. 14, the Cluster-based algorithm characterizes a property of maximum energy consumption. Compared to RTA, TOBPA's index is almost equivalent to RTA's. The difference between them only refers to the scenarios where partial Range-Short nodes are scheduled in step 2 of TOBPA.Therefore, TOBPA saves more energy than RTA.

\section{Conclusion}

We propose a clustered structure with MEs for reducing and balancing energy consumption in WSNs. RPs and R-RPs are designed by geometrical relationship for every cluster to achieve the energy consumption optimization, respectively. Based on the transmission model, we define utility function and apply DMUR for RTA and TOBPA to optimize the selection of RNs, which store and forward data for energy efficiency. Except for delay, RTA and TOBPA are established to achieve optimal energy consumption as well as energy balance with cooperation among MEs. However, we also acknowledge that shortcomings of mobility exist, which definitely incurs latency problem for data collection and energy resource limitations for MEs. In the future, we will plan to exploit the technology of mobile edge computing to solve above mentioned problems. Besides, we will focus on other factors by considering other factors, such as channel information, communication reliability, and so on.

\section{References}

[1] G. Niayesh, B. K. Abu, H. S. Z. Mohd, P. A. Hosseingholi, B. S. Ashfaq, “ Collaborative mobile sink sojourn time optimization scheme for cluster-based wireless sensor networks, ” IEEE Sensors Journal, vol. 18, no. 16, pp. 6669-6676, Aug. 2018. Article (CrossRef Link)

[2] Zhuang, W., Chen, Y., Su, J., Wang, B., \& Gao, C., "Design of human activity recognition algorithms based on a single wearable IMU sensor,” International Journal of Sensor Networks, vol.30, no.3, pp.193-206, 2019. Article (CrossRef Link)

[3] Bo Y., Xuetao W,

"Communication-Efficient Data Aggregation Tree Construction for Complex Queries in IoT Applications.” IE EE Internet of Things Journal, vol.6, no.2, pp.3352-3363, 2019. Article (CrossRef Link)

[4] J. Su, Z. Sheng, V. C.M. Leung, Y. Chen, "Energy efficient tag identification algorithms for RFID: survey, motivation and new design,” IEEE Wireless Communications, vol. 26, no. 3, pp. 118-124, 2019. Article (CrossRef Link)

[5] Jian Zhang, Jian Tang, Feng Wang, "Cooperative Relay Selection for Load Balancing With Mobility in Hierarchical WSNs: A Multi-Armed Bandit Approach.” IEEE Access, vol.8, pp. 18110-18122, 2020. Article (CrossRef Link)

[6] Shimng He, Kun Xie, Kexin Xie, Chuan Xu, Jin Wang, "Interference-aware Multi-source Trans mission in Multi-radio and Multi-channel Wireless Network.” IEEE Systems Journal, vol.13, no.3, pp. 2507-2518, 2019. Article (CrossRef Link)

[7] C. Sha, D. Song, R. Yang, H. Gao, H. Huang, “A Type of Energy-Balanced Tree Based Data Collection Strategy for Sensor Network With Mobile Sink,” IEEE Access, vol. 7, pp. 85226-85240, Jun. 2019. Article (CrossRef Link)

[8] T.Wang, Y. Li, G. Wang, J. Cao, M. Z. A. Bhuiyan, W. Jia, "Sustainable and Efficient Data Collection from WSNs to Cloud," IEEE Transactions on Mobile Computing, vol. 4, no. 2, pp. 252-262, Mar. 2019. Article (CrossRef Link)

[9] Mohammed Kaddi, Khelifa Benahmed and Mohammed Omari, “An Energy-Efficient Protocol Using an Objective Function \& Random Search with Jumps for WSN,” Computers, Materials \& Continua, Vol. 58, No. 3, pp. 603-624, 2019. Article (CrossRef Link) 
[10] G. Xu, E. C. H. Ngai, J. Liu, "Ubiquitous Transmission of Multimedia Sensor Data in Internet of Things,” IEEE Internet of Things Journal, vol. 5, no. 1, pp. 403- 414, Feb. 2018. Article (CrossRef Link)

[11] K. Liu, J. Peng, L. He, J, Pan, S. Li, M. Ling, Z. Huang, “An Active Mobile Charging and Data Collection Scheme for Clustered Sensor Networks,” IEEE Transactions on Vehicular Technology, vol. 68, no. 5, pp. 5100-5113, Mar. 2019. Article (CrossRef Link)

[12] Y. Liu, K.Y. Lam, S. Han, Q. Chen, "Mobile data gathering and energy harvesting in rechargeable wireless sensor networks,” Information Sciences, vol. 482, pp. 189-209, May, 2019. Article (CrossRef Link)

[13] J. Zhang, T. Wang, J. Tang, “Optimising rendezvous-based data collection for target tracking in WSNs with mobile elements,” Int. J. Sensor Networks, vol. 30, no. 4, pp. 218-230, Jul. 2019. Article (CrossRef Link)

[14] K. T.M. Tran, S. H. Oh, "UWSNs: A round-based clustering scheme for data redundancy resolve," Int. J. Distrib. Sensor Netw., vol.10, no.4, pp. 1-6, Apr. 2014. Article (CrossRef Link)

[15] G. Anastasi, M. Conti, M. D. Francescoa, A. Passarella, "Energy conservation in wireless sensor networks: A survey,” Elsevier Ad Hoc Netw., vol. 7, no. 3, pp. 537-568, May 2009. Article (CrossRef Link)

[16] M. Krishnan, S. Yun, Y. M. Jung, "Enhanced clustering and ACO-based multiple mobile sinks for efficiency improvement of wireless sensor networks," Computer Networks, vol.160, pp. 33-40, Sept. 2019. Article (CrossRef Link)

[17] Y. Miao, Z, Sun, N, Wang, Y, Cao, H, Cruickshank, “Time Efficient Data Collection With Mobile Sink and vMIMO Technique in Wireless Sensor Networks,” IEEE Systems Journal, vol. 12, no. 1 , pp. 639-647,Mar. 2018. Article (CrossRef Link)

[18] D. Ebrahimi , S. Sharafeddine , P.H. Ho , C. Assi, "UAV-Aided Projection-Based Compressive Data Gathering in Wireless Sensor Networks,” IEEE Internet of Things Journal, vol. 6, no. 2, pp. 1893-1905, Apr. 2019. Article (CrossRef Link)

[19] R. Yarinezhad, "Reducing delay and prolonging the lifetime of wireless sensor network using efficient routing protocol based on mobile sink and virtual infrastructure,” Ad Hoc Networks, vol. 84, pp. 42-55, Mar. 2019. Article (CrossRef Link)

[20] J. Su, Z. Sheng, A. Liu, Z. Fu, and Y. Chen, "A time and energy saving based frame adjustment strategy (TES-FAS) tag identification algorithm for UHF RFID systems," IEEE Transactions on Wireless Communications, pp. 1-13, 2020. Article (CrossRef Link)

[21] Z. Xu, L, Chen, C. Chen, X, Guan, “Joint Clustering and Routing Design for Reliable and Efficient Data Collection in Large-Scale Wireless Sensor Networks,” IEEE Internet of Things Journal, vol. 3, no. 4, pp. 520-532, Aug. 2016. Article (CrossRef Link)

[22] M. Yang, S. Wang, A. Abdelal, Y. Jiang, Y. Kim, “An Improved Multi-Layered Architecture and its Rotational Scheme for Large-Scale Wireless Sensor Networks,” in Proc. of 4th IEEE Conference of Consumer Communications and Networking, Las Vegas, NV, USA, pp.855-859, 11-13 Jan. 2007. Article (CrossRef Link)

[23] J. Zhang , S.J. Liu , P.W. Tsai , F.M. Zou , X.R. Ji , "Directional virtual backbone based data aggregation scheme for wireless visual sensor networks,” PLoS One, vol. 13 , no. 5, e0196705, May. 2018. Article (CrossRef Link)

[24] Thejaswini M., Bong Jun Choi, "Weighted Adaptive Opportunistic Scheduling Framework for Smartphone Sensor Data Collection in IoT,” KSII Transactions on Internet and Information Systems. Vol. 13, No. 12, pp.5805-5825, December 31, 2019. Article (CrossRef Link)

[25] M. Dong, K. Ota, A. Liu, "RMER: Reliable and Energy-Efficient Data Collection for Large-Scale Wireless Sensor Networks,” IEEE Internet of Things Journal, vol. 3, no. 4, pp. 511-519, Jan. 2016. 2019. Article (CrossRef Link)

[26] F. Tashtarian , M. H. Y. Moghaddam, K. Sohraby, S. Effati, “On Maximizing the Lifetime of Wireless Sensor Networks in Event-Driven Applications With Mobile Sinks,” IEEE Transactions on Vehicular Technology, vol. 64, no. 7, pp. 3177-3189, Jul. 2015. Article (CrossRef Link) 
[27] C. Zhan, Y. Zeng, R. Zhang, "Energy-Efficient Data Collection in UAV Enabled Wireless Sensor Network,” IEEE Wireless Communications Letters, Vol. 7, no. 3, pp. 328-331, Jun. 2018. Article (CrossRef Link)

[28] D. Izadi, S. Ghanavati, J. Abawajy, T. Herawan, “An alternative data collection scheduling scheme in wireless sensor networks,” Computing, vol. 98, no. 12, pp. 1287-1304, Dec. 2016. Article (CrossRef Link)

[29] N. Gharaei, K. A. Bakar, S. Z. M. Hashim, A. H. Pourasl, S. A. Butt, "Collaborative Mobile Sink Sojourn Time Optimization Scheme for Cluster-Based Wireless Sensor Networks,” IEEE Sensors Journal, vol. 18, no. 16, pp. 6669-6676, Aug. 2018. Article (CrossRef Link)

[30] W. B. Heinzelman, A. P. Chandrakasan, H. Balakrishnan, “An application-specific protocol architecture for wireless microsensor networks,” IEEE Trans. Wireless Commun., vol. 1, no. 4, pp. 660-670, Oct. 2002. Article (CrossRef Link)

[31] R. Zhang, J. Pan, D. Xie, F. Wang, "NDCMC: A Hybrid Data Collection Approach for Large-Scale WSNs Using Mobile Element and Hierarchical Clustering," IEEE Internet of Things Journal, vol. 3, no. 4, pp. 533-543, Aug. 2016. Article (CrossRef Link)

[32] K. Haseeb, K. A. Bakar, A. H. Abdullah, and T. Darwish, "Adaptive energy aware cluster-based routing protocol for wireless sensor networks,” Wireless Networks, vol. 23, no. 6, pp. 1953-1966, Aug. 2017. Article (CrossRef Link)

[33] R. Deng, S. He, J. Chen, “An Online Algorithm for Data Collection by Multiple Sinks in Wireless-Sensor Networks,” IEEE Transactions on Control of Network Systems, vol. 5, no. 1, pp. 93-104, Mar. 2018. Article (CrossRef Link)

[34] Jin Wang, Yu Gao, Xiang Yin, Feng Li and Hye-Jin Kim, “An Enhanced PEGASIS Algorithm with Mobile Sink Support for Wireless Sensor Networks,” Wireless Communications and Mobile Computing, vol.8, pp.1-9, 2018. Article (CrossRef Link)

[35] G. Xing, M. Li, T. Wang, W. Jia, J. Huang, "Efficient rendezvous algorithms for mobility-enabled wireless sensor networks,” IEEE Tran. Mobile Comput., vol. 11, no. 1, pp. 47-60, Jan. 2012. Article (CrossRef Link)

[36] H. Salarian, K. W. Chin, F. Naghdy, "An Energy-Efficient Mobile-Sink Path Selection Strategy for Wireless Sensor Networks," IEEE Transactions on Vehicular Technology, vol. 63, no.5, pp.2407-2419, Jun. 2014. Article (CrossRef Link)

[37] A. Wichmann, T. Korkmaz, A. S. Tosun, "Robot Control Strategies for Task Allocation with Connectivity Constraints in Wireless Sensor and Robot Networks,” IEEE Transactions on Mobile Computing, vol. 17, no. 6,pp. 1429-1441, Jun. 2018. Article (CrossRef Link)

[38] J. Wang, J. Cao, S. Ji, and J. H. Park, “Energy-efficient cluster-based dynamic routes adjustment approach for wireless sensor networks with mobile sinks,: The Journal of Supercomputing, vol. 73, no. 7, pp. 3277-3290, Jul. 2017. Article (CrossRef Link)

[39] W. Liu , J. Fan, "Efficient rendezvous schemes for mobile collector in wireless sensor networks with relay hop constraint,” Ad hoc \& Sensor Wireless Networks, vol. 30, no. 3/4, pp. 219-240, Jan. 2016.

https://www.oldcitypublishing.com/journals/ahswn-home/ahswn-issue-contents/ahswn-volume-3 0-number-3-4-2016/ahswn-30-3-4-p-219-240/

[40] F. Yan , X. Zhang , L. Tao, H. Zhang, "Network Coding-Based Flooding with a Mobile Sink in Low-Duty-Cycle Wireless Sensor Networks,” IEEE Tran. Mobile Comput., VOL. 18, NO. 8, pp. 1857-1869, AUG. 2019. Article (CrossRef Link)

[41] M. Abbas, S. N. Vahid, A. Omid, “An Aging-aware Energy-efficient Data Collection Approach in Wireless Sensor Networks using Multiple Mobile Sinks,” in Proc. of 27th Iranian Conference on Electrical Engineering (ICEE), Yazd, Iran, Iran,30 April-2 May 2019. Article (CrossRef Link)

[42] S. Yang, U. Adeel, Y. Tahir, J, A. McCann, "Practical Opportunistic Data Collection in Wireless Sensor Networks with Mobile Sinks,” IEEE Transactions on Mobile Computing,vol.16, no. 5, pp. 1420-1433, May 2017. Article (CrossRef Link)

[43] V. Kavidha, S. Ananthakumaran, "Novel energy-efficient secure routing protocol for wireless sensor networks with Mobile sink,” Peer-to-Peer Networking and Applications, vol. 12, no. 4, pp. 881-892, Jul. 2019. Article (CrossRef Link) 

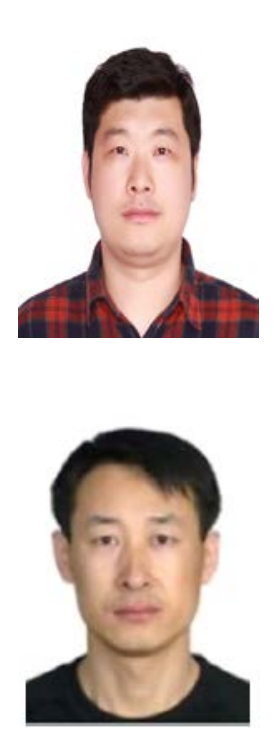

JIAN ZHANG received his MS degree and Ph.D. degree in Applied Mathematics from Liaoning University and in Pattern Recognition and Intelligent Systems from Northeastern University, China, in 2008 and 2012, respectively. Since 2012, he has been working at Nanjing University of Information Science and Technology, China. His research interests include wireless sensor networks, mobile computing and target tracking.

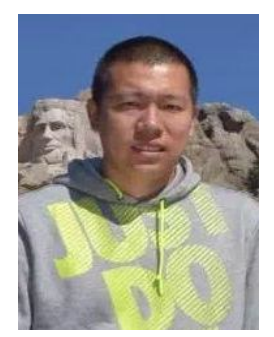

Jian Tang received the Ph.D. degree from Northeastern University, Shenyang, China, in 2012, in control theory and control engineering. He is currently a Professor with Beijing University of Technology. His current research interests are small sample data intelligent modeling and intelligent control of complex industrial process. He has made research on soft measuring industrial process parameters based on mechanical frequency spectrum data about 10 years. His research also focuses on modeling and control of municipal solid waste incineration process since 2017. He has published the relative SCI/EI papers more than 50 ones.

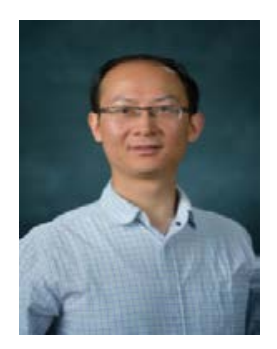

ZHONGHUI WANG received his MS degree in Computer Science and Information from University of Mississippi, MS, USA, in 2016. Currently, he is pursuing a Ph.D. degree with University of Mississippi, USA.His current research interests are wireless visual sensor networks and internet of things.

FENG WANG (S’07-M'13) received his Bachelor of Engineering and Master of Engineering (both in Computer Science) from Tsinghua University, Beijing, China, in 2002 and 2005, respectively. He received his PhD (in Computer Science) from Simon Fraser University, Burnaby (Metro-Vancouver), British Columbia, Canada, in 2012. He is a recipient of the Chinese Government Scholarship for Outstanding Self-financed Students Studying Aboard (2009) and IEEE ICME Quality Reviewer Award (2011). he serves as Program Vice Chair in International Conference on Internet of Vehicles (IOV) 2014, and serves as TPC co-chair in IEEE International Conference on Communications in China (ICCC) 2014 for Symposium on Wireless Networking and Multimedia. He also serves as TPC member in various international conferences such as such as IEEE/ACM IWQoS, ACM Multimedia, IEEE ICC, IEEE GLOBECOM, IEEE CloudCom and IEEE ICME.His research interests are mainly in computer networking area, in particular, wireless sensor/mesh networks, peer-to-peer networks, socialized content sharing, cyber-physical systems, internet of things, cloud computing, big data and distributed computing. 